\title{
"ESTRATEGIAS DE APRENDIZAJE DE LOS ESTUDIANTES DE LA FACULTAD DE ENFERMERÍA DE LA UNIVERSIDAD NACIONAL SAN LUIS GONZAGA DE ICA, Y SU RELACIÓN CON EL RENDIMIENTO ACADÉMICO, CICLO 2012-II"
}

\author{
Zonia Quispe Quispe ${ }^{1, a, d}$, Deyda García Pérez, ${ }^{2, c}$, Yolanda Chinarro de Pun ${ }^{3, b, c}$
}

\footnotetext{
${ }^{1}$ Facultad de Enfermería de la Universidad Nacional San Luis Gonzaga de Ica, Doctora en Salud Pública; ${ }^{2}$ Hospital Santa María del socorro Ica. Ica, Perú ${ }^{3}$ Hospital Regional de Ica. Ica, Perú.

a Licenciada en Enfermería, ${ }^{b}$ Licenciada en Nutrición, ${ }^{\circ}$ Magíster en Educación con Mención en Administración y Planificación de la Educación Superior. ${ }^{\mathrm{d}}$ Doctora en Salud Pública.
}

\section{RESUMEN}

Objetivo: Analizar la relación entre las estrategias de aprendizaje y el rendimiento académico de los estudiantes de la Facultad de Enfermería de la Universidad Nacional San Luis Gonzaga de Ica Perú Material y Métodos: Se adaptó el cuestionario ACRA, validado y confiabilizado mediante alfa de Cronbach $(0,841)$, utilizándose las actas consolidadas del 2012-II. El estudio fue exploratorio, descriptivo, correlacional, aplicado a 139 estudiantes distribuidos del I al VIII Ciclo de estudios. La hipótesis de investigación se probó mediante Ji cuadrada para 95\% de confianza. Resultados: Se encontró que $41,7 \%$ de los estudiantes utilizan estrategias de apoyo al procesamiento, $36 \%$ de recuperación, $14,4 \%$ de adquisición y $7,9 \%$ de codificación. El 58,4\% de los estudiantes que tienen rendimiento académico inferior al promedio utilizan la estrategia de procesamiento, 43,7\% de los que tienen rendimiento alto y $29 \%$ de los que tienen rendimiento promedio también. $40 \%$ de los estudiantes con rendimiento promedio usan estrategias de recuperación de la información para su aprendizaje. Mediante Ji cuadrada se probó que no existe relación significativa entre las estrategias y el rendimiento académico. Las medias aritméticas indican los índices para las estrategias de procesamiento $(3,04)$, similar a las de recuperación $(3,00)$, ligeramente superior a las estrategias de adquisición $(2,84)$ y codificación $(2,75)$ en una escala máxima de 4.0 . Conclusiones: No existe relación significativa entre estrategias de aprendizaje y el rendimiento académico, las estrategias de aprendizaje más predominantes son: 41,7\%, 36\%, 14,4\% y 7,9\% correspondiéndole a: procesamiento, recuperación de la información, adquisición y codificación respectivamente.

Palabras clave: Estrategia, Aprendizaje, rendimiento académico, estudiantes de enfermería.

\section{SUMMARY}

Objective: To analyze the relationship between learning strategies and academic performance of students in the Faculty of Nursing at San Luis Gonzaga National University of Ica - Peru. Material and Methods: ACRA questionnaire was adapted and validated using Cronbach's alpha confiabilizado (0.841), using the 2012-II consolidated proceedings. The study was exploratory, descriptive, correlational, applied to 139 students from I to VIII distributed cycle studies. The research hypotheses were tested by chi-square for $95 \%$ confidence. Results: We found that $41.7 \%$ of students use strategies to support the processing, 36\% recovery, $14.4 \%$ and $7.9 \%$ acquisition coding. $58.4 \%$ of students with below average academic performance processing strategy used, $43.7 \%$ of those with high performance and $29 \%$ of those with average too. $40 \%$ of students with average recovery strategies use information for learning. By Chi square is proved that there is no significant relationship between strategies and academic performance. The indices indicate arithmetic means for processing strategies (3.04), similar to recovery (3.00), slightly higher than acquisition strategies (2.84) and encoding (2.75) on a scale maximum of 4.0. Conclusions: There is no significant relationship between learning strategies and academic performance, learning strategies are most prevalent: $41.7 \%, 36 \%, 14.4 \%$ and $7.9 \%$ correspondiéndole to: processing, information retrieval, acquisition and coding respectively.

Keywords: Strategy, Learning, academic performance, student nurses. 


\section{INTRODUCCIÓN}

En la actualidad estamos viviendo acelerados cambios que involucran todos los aspectos de la vida humana donde se incluye la formación profesional. Los conocimientos se vienen multiplicando y profundizando cada día en una forma vertiginosa, de acuerdo al avance de la ciencia y tecnología, tal que surgen necesidades que exigen conocimientos transdisciplinarios. Frente a este avance en la formación, la educación tiene como reto fundamental formar hombres y mujeres con actitudes positivas, con capacidades de buscar informaciones actualizadas, seleccionar, sistematizar, utilizar nuevos conocimientos, dentro de un proceso que debe verse acompañado necesariamente de una toma de conciencia por parte del estudiante y de los docentes, de cómo es que aprende el estudiante y qué método, estrategia o estilo de aprendizaje utiliza para mejorar sus conocimientos. Es en este contexto que surge el interés por identificar, las estrategias de aprendizaje idóneas a fin de poder instrumentar métodos de enseñanza eficaces para lograr un rendimiento académico significativo, y despertar el interés del estudiante no solo en las asignaturas de especialidad, sino también en las de formación general.

En cuanto a las estrategias de aprendizaje (1), lo definió como: Técnicas de aprendizaje andragógicos y que los recursos varían de acuerdo con los objetivos, contenidos del estudio y aprendizaje de la formación previa de los participantes, posibilidades, capacidades y limitaciones personales de cada quien. Las estrategias van desde las simples habilidades de estudio, como el subrayado de la idea principal, hasta los procesos de pensamiento complejo, como usar las analogías para relacionar el conocimiento previo con la nueva información. Una primera aproximación a las estrategias de aprendizaje nos remite a la diferenciación entre estrategias impuestas e inducidas, principalmente referidas al estudio de textos escolares. Las primeras son impuestas por el profesor o programador de textos al realizar modificaciones 0 manipulaciones en el contenido o estructura del material de aprendizaje. Las estrategias inducidas se vinculan con el entrenamiento de los estudiantes para manejar directamente y por sí mismos procedimientos que les permitan aprender con éxito. Es decir, las estrategias impuestas son elementos didácticos que se intercalan, como resúmenes, preguntas de reflexión, ejercicios, autoevaluaciones, etc., mientras que las estrategias inducidas son aportaciones, como el auto-interrogatorio, la elaboración, la repetición y la imaginación, los cuales son desarrollados por el estudiante y constituyen sus propias estrategias de aprendizaje. Los dos tipos de estrategias: instruccionales (impuestas) y de aprendizaje (inducidas), son estrategias cognoscitivas, involucradas en el procesamiento de la información a partir de textos, que realiza un lector, aun cuando en el primer caso el énfasis se hace en el material $y$ el segundo en el aprendiz (2). Utilizando para el estudio el instrumento de evaluación ACRA, siendo los siguientes:

- ADQUISICIÓN de la información, con estrategias atencionales (exploración y fragmentación) y estrategias de repetición.

- CODIFICACIÓN de la información: estrategias de nemotecnización, estrategias de elaboración y estrategias de organización.

- RECUPERACIÓN de la información: estrategias de búsqueda en la memoria (búsqueda de codificaciones y de indicios), estrategias de generación de Respuesta (planificación y preparación de la respuesta escrita).

- APOYO al procesamiento, se divide en: estrategias metacognitivas (autoconocimiento y de automanejo), estrategias afectivas (autoinstrucciones, autocontrol, y contradistractoras), sociales (interacciones sociales), y motivacionales (motivación intrínseca, motivación extrínseca y motivación de escape) (3). 
Teniendo en consideración tales aportes se ha observado que los estudiantes de la Facultad de Enfermería de la Universidad San Luis Gonzaga de Ica, utilizan indiferentemente las estrategias de aprendizaje, y no se relaciona significativamente con su rendimiento académico, situación que se conoce en la presente investigación.

El Objetivo fue analizar la relación entre las estrategias de aprendizaje y el rendimiento académico de los estudiantes de la Facultad de Enfermería de la Universidad Nacional San Luis Gonzaga de Ica - Perú.

\section{MATERIAL Y MÉTODOS}

La investigación se llevó a cabo en la Facultad de Enfermería de la Universidad Nacional San Luis Gonzaga de Ica, ubicada en la Ciudad Universitaria, AV Los Maestros $\mathrm{S} / \mathrm{N}$, Ica - Perú. Muestra de estudio estuvo compuesta por 139 estudiantes distribuidos del I al VIII ciclo de estudios correspondiente al año académico 2012-II. El estudio corresponde al tipo de investigación no experimental, sin manipulación de variables, de nivel descriptivo, correlacional, de diseño transversal, que analiza las variables: Estrategias de Aprendizaje y rendimiento Académico. Para realizar la evaluación y medición de las estrategias utilizadas por los alumnos se empleó el cuestionario A.C.R.A. (Román y Gallego, 1994). Dicho instrumento autoevaluativo refleja puntuaciones referidas al empleo de estrategias referidas a 4 escalas generales: Adquisición (estrategias atencionales y de repetición), Codificación (nemotecnias, organización y elaboración), Recuperación de la información (estrategias de búsqueda y de generación de respuesta), y Apoyo al procesamiento (estrategias socioafectivas y metacognitivas).

Rendimiento académico se observó a partir del consolidado de las actas finales de las asignaturas. La validez se determinó mediante índice de acuerdos y desacuerdos por 3 expertos cuyo índice fue de 0.83 , y la confiabilidad se determinó mediante Alfa de
Cronbach en dos pruebas sucesivas con 10 estudiantes cuyo índice fue 0,841.

\section{RESULTADOS}

Se puede apreciar en la tabla 1, que el $41,7 \%$ de los estudiantes utilizan como estrategias de aprendizaje las del apoyo al procesamiento de la información, seguida por las estrategias de recuperación de la información (36\%), una menor proporción de estudiantes utilizan las estrategias de adquisición $(14,4 \%)$ y de codificación de la información $(7,9 \%)$.

Tabla 1. Predominancia de las Estrategias de Aprendizaje

\begin{tabular}{lcc}
\hline \multicolumn{1}{c}{ Estrategias } & Frecuencia & \% Frecuencia \\
\hline Adquisición & 20 & 14,4 \\
Codificación & 11 & 7,9 \\
Recuperación & 50 & 36,0 \\
Procesamiento & 58 & 41,7 \\
TOTAL & 139 & $100 \%$ \\
\hline
\end{tabular}

En cuanto a las estrategias de aprendizaje, en la tabla 2 se muestra las estrategias según el ciclo de estudios. Se puede apreciar que en los estudiantes del I ciclo prevalecen las estrategias de adquisición de la información, en los estudiantes del II, IV y V ciclo prevalecen las estrategias de recuperación de la información, y en los estudiantes del III, VI, VII y VIII ciclo prevalecen las estrategias de apoyo al procesamiento de la información. En los últimos ciclos Vi, VII y VIII prevalecen las estrategias de apoyo al procesamiento de la información, es decir adquieren información mediante estrategias metacognitivas (autoconocimiento y automanejo), estrategias afectivas (autoinstrucción, autocontrol y contradictorias), sociales y motivacionales.

Tabla 2. Estrategia de Aprendizaje según ciclo de estudios

\begin{tabular}{ccccc}
\hline Ciclo & Adquisición & $\begin{array}{c}\text { Codificac } \\
\text { ión }\end{array}$ & $\begin{array}{c}\text { Recuperaci } \\
\text { ón }\end{array}$ & $\begin{array}{c}\text { Procesa } \\
\text { miento }\end{array}$ \\
\hline I & 2,917 & 2,75 & 2,89 & 2,9 \\
II & 2,80 & 2,83 & 3,08 & 3,0 \\
III & 2,53 & 2,90 & 2,82 & 3,0 \\
IV & 2,995 & 2,91 & 3,22 & 3,1 \\
V & 2,778 & 2,71 & 3,06 & 3,0 \\
VI & 2,634 & 2,50 & 2,72 & 2,9 \\
VII & 3,005 & 2,77 & 2,98 & 3,2 \\
VIII & 3,00 & 2,87 & 3,10 & 3,2 \\
Prom & 2,84 & 2,75 & 2,99 & 3,04 \\
\hline
\end{tabular}


En la tabla 3 se puede apreciar que el rendimiento académico promedio de los estudiantes encuestados del VIII Ciclo es de 14,36, del sétimo ciclo 14,1, del II Ciclo es de 13,58, del II Ciclo 13,29, del I Ciclo 13,28, del IV y VI ciclo es de 13,01, y el rendimiento académico promedio de los estudiantes del $\mathrm{V}$ ciclo es de 12,65. El rendimiento académico promedio del I al VIII es $\bar{X}=13,41$

\section{Tabla 3. Rendimiento Académico (Promedio)}

\begin{tabular}{lccccc}
\hline \multicolumn{1}{c}{ Ciclo } & $\overline{\boldsymbol{X}}$ & $\overline{\boldsymbol{X}}+\boldsymbol{D} \boldsymbol{S}$ & $\overline{\boldsymbol{X}}-\boldsymbol{D S}$ & $\mathbf{n}$ & $\mathbf{D S}$ \\
\hline I & 13,28 & 6 & 8 & 43 & 2,01 \\
II & 13,58 & 5 & 8 & 69 & 2,42 \\
III & 13,29 & 1 & 4 & 42 & 3,00 \\
IV & 13,01 & 6 & 5 & 42 & 2,13 \\
V & 12,65 & 7 & 7 & 40 & 2,01 \\
VI & 13,01 & 3 & 6 & 38 & 3,28 \\
VII & 14,10 & 2 & 3 & 36 & 2,11 \\
VIII & 14,36 & 6 & 7 & 33 & 0,99 \\
TOTAL & 13,41 & 36 & 48 & 139 & ---- \\
\hline
\end{tabular}

La tabla 4 muestra el resultado que relaciona las estrategias de aprendizaje que utilizan los estudiantes encuestados, de acuerdo al rendimiento académico que tienen. Se puede apreciar que el $58,4 \%$ de los estudiantes encuestados que tienen un rendimiento académico inferior a la media aritmética (promedio) practican las estrategias de procesamiento de la información para su aprendizaje, y $25 \%$ utilizan estrategias de codificación. $\quad 40 \%$ de los estudiantes encuestados que tienen rendimiento académico promedio, practican las estrategias de recuperación de la información, para su aprendizaje, 29\% utilizan estrategias de procesamiento. $43,7 \%$ de los estudiantes encuestados que tienen rendimiento académico superior al promedio, practican las estrategias de procesamiento de la información para su aprendizaje, y 39,6\% utilizan estrategias de recuperación.
Tabla 4. Estrategias de aprendizaje según el rendimiento académico

\begin{tabular}{cccccccc}
\hline Estrategia & \multicolumn{6}{c}{ Rendimiento Académico } \\
\cline { 2 - 7 } & $\begin{array}{c}\text { Inferior al } \\
\text { promedio }\end{array}$ & Promedio & $\begin{array}{c}\text { Superior al } \\
\text { Promedio }\end{array}$ \\
\hline Adquisición & 4 & $\%$ & $f$ & $\%$ & $f$ & $\%$ \\
Codificación & 2 & 5,5 & 6 & 11 & 3 & 6,3 \\
Recuperación & 9 & 25.0 & 22 & 40 & 19 & 39.6 \\
Procesamiento & 21 & 58.4 & 16 & 29 & 21 & 43.7 \\
TOTAL & 36 & 100 & 55 & 100 & 48 & 100 \\
\hline
\end{tabular}

Finalmente se determinaron los datos generales de la muestra de estudios: Se puede apreciar que el $57,6 \%$ de los estudiantes encuestados tienen entre 18 y 20 años de edad, 19,4\% entre 21 y 23 años, $15,8 \%$ entre 15 y 17 años y 7,2 tienen entre 24 a 29 años. Se puede apreciar que $87,8 \%$ de los estudiantes encuestados son de sexo femenino y $12,2 \%$ de sexo masculino. Se observa que $66.2 \%$ de los estudiantes proceden de colegios nacionales y $33,8 \%$ de colegios particulares.

\section{DISCUSIÓN.}

En la investigación se han identificado cuatro niveles de estrategias de aprendizaje: Procesamiento $(3,04)$, recuperación $(3,0)$, adquisición $(2,84)$ y codificación $(2,75)$, de acuerdo a su utilización por parte de los estudiantes, si bien se aprecia índices similares en las dos primeras estrategias (Procesamiento y recuperación), se evidencia una utilización diferenciada de estos con las estrategias de adquisición y codificación. Este hallazgo significa que los estudiantes de enfermería mayormente adquieren información mediante estrategias metacognitivas (autoconocimiento y de automanejo), estrategias efectivas (autoinstrucciones, autocontrol y contradistractoras), sociales y motivacionales, paralelamente adquieren información mediante estrategias de búsqueda en la memoria, y de generación de respuesta (planificación y preparación de la respuesta escrita), esta característica de los estudiantes 
de enfermería estaría contrastando con los hallazgos de Loret de Mola (4) quien para los estudiantes de educación y ciencias humanas de la Universidad Peruana "Los Andes", encontró que utilizaban mayormente la estrategia de codificación y utilizan menos las estrategias de apoyo al procesamiento, lo cual nos grafica el uso de estrategias en carreras de naturaleza diferente. por otra parte, Fuente (5) asegura que la utilización de estrategias de aprendizaje no son significativamente diferentes en las diferentes carreras de la universidad, lo cual es bastante aproximado a nuestro caso.

Por otra parte, Camarero (6) en diferentes especialidades de la Universidad de Oviedo, encontró mayor uso de la escala de apoyo al procesamiento de la información, lo cual es concurrente con nuestro estudio. En cuanto al rendimiento académico, el nivel que encontramos es regular (promedio global 13,41), en contraste con el nivel encontrado por Loret de Mola (4), que fue bueno, tal vez esto se pueda explicar debido a carreras diferentes en ambos estudios, $y$ a las dificultades de la naturaleza de las asignaturas. Los resultados de rendimiento encontrados nos señalan una tendencia a que conforme se avanzan los ciclos de estudio, se incrementa el índice del rendimiento (I ciclo 13,28, VIII ciclo 14,36).

La prueba de la hipótesis mediante $\mathrm{Ji}$ cuadrada, informa que no hay relación estadística significativa entre las estrategias de aprendizaje y el rendimiento académico, se ha encontrado un valor positivo de $x^{2} 8,25$, menor a $x^{2} 12,59$ para $95 \%$ de confianza, que se puede explicar por los índices aproximados encontrados en las cuatro estrategias $(3,04-3,00-2,84-2,75)$ y en el rendimiento académico de los estudiantes del I al VIII ciclo $(12,65$ - 14,36). Los resultados de nivel regular en el rendimiento académico, demuestra que existe la necesidad de mejorar, con uno de sus factores que puede ser la utilización adecuada de estrategias cognitivas y metacognitivas, según Beltrán
(7), las estrategias de aprendizaje sirven para mejorar la calidad del rendimiento académico. Román (8) considera que las estrategias de aprendizaje son el camino para desarrollar destrezas, actitudes por medio de contenidos y métodos, Monereo (1) afirma que las estrategias de aprendizaje son procesos de toma de decisiones conscientes e intencionales que elige el estudiante para recuperar conocimientos.

\section{CONCLUSIONES}

1. No existe relación significativa entre las estrategias de aprendizaje y el rendimiento académico.

2. Las estrategias de aprendizaje más predominantes son la de procesamiento $(41,7 \%)$ y recuperación de la información $(36 \%)$, y las menos predominantes la adquisición $(14,4 \%)$ y la codificación $(7,9 \%)$

3. El nivel del rendimiento académico de los estudiantes es regular ( $\bar{X} 13,41 \%)$.

\section{Correspondencia:}

Dra. Zonia Quispe

Correo electrónico: zoniaquispe@hotmail.com

\section{REFERENCIAS BIBLIOGRÁFICAS}

1. Monereo, C. et al. Estrategias de enseñanza y aprendizaje. Barcelona: Editorial GRAO 9na. Edición. 2001.

2. Aguilar D. Estrategias de aprendizaje para la comprensión de textos académicos en prosa en. Perfiles educativos. Lima, Perú. 1988.

3. Román, J.M. ACRA. Escalas de estrategias de aprendizaje. Madrid, España. TEA. 1994.

4. Loret de Mola. Estilos y estrategias de aprendizaje en el rendimiento académico de los estudiantes de la Universidad Peruana "Los Andes" de Huancayo Perú. Revista Estilos de Aprendizaje No 8. Vol. 8oct. 2011. 
5. Fuente, J. Factores condicionantes de las Estrategias de Aprendizaje y del Rendimiento Académico en alumnos universitarios, a través del ACRA. Investigación del Dpto. de Psicología Evolutivo y de la Educación. Facultad de Humanidades y Ciencias de la Educación. Universidad de Almería, España. 1994.

6. Camarero F. Estilos y estrategias de aprendizaje en estudiantes universitarios. Psicoterma. Vol. 12, № 4, pp. 515-522. Oviedo, España. 2000.

7. Beltrán, J. Psicología de la Educación. Ed. Eudema. Madrid, España. 1993.
8. Román M. Revista Enfoques educacionales Vol. 2 El Currículo como desarrollo de procesos cognitivos y afectivos. Universidad de Chile. 200. Consultado el 05-04-07 en:

http://rehue.esociales.uchile.cl/publicacio n es/enfoques/04/idn.04.htm

Recibido: 07/02/14

Aprobado para Publicación: 16/05/14. 